\title{
Continuous smearing of Wilson Loops.
}

\section{Robert Lohmayer*}

Rutgers University, Department of Physics and Astronomy, Piscataway, NJ 08854

E-mail: Lohamyeraphysics.rutgers.edu

\section{Herbert Neuberger ${ }^{\dagger *}$}

Rutgers University, Department of Physics and Astronomy, Piscataway, NJ 08854

E-mail: neubergephysics.rutgers.edu

Continuum smearing was introduced in section 4.1 of JHEP03, 064 (2006) as a meaningful continuum analogue of the well known set of lattice techniques by the same name. Here we apply continuous smearing in continuous space-time to Wilson loops in order to clarify what it does in the context of field theory and also in the context of the loop calculus of the Makeenko-Migdal equation.

XXIX International Symposium on Lattice Field Theory

July 10-16, 2011

Squaw Valley, Lake Tahoe, California

\footnotetext{
* Research supported in part by the DOE, grant nr. DE-FG02-01ER41165

${ }^{\dagger}$ Speaker.

₹esearch supported in part by the DOE, grant nr. DE-FG02-01ER41165
} 


\section{What is smearing?}

In standard form, smearing is a map $S\left[\phi_{0}(x)\right]=\phi_{s}(x)$ of general structure $\partial_{s} \phi_{s}(x)=F\left[\phi_{s}(x)\right]$ where $F[$.$] has some locality property and an initial condition is supplied at s=0 . \phi_{0}(x)$ denotes a set of fields defined on a smooth manifold and $x$ is a point on that manifold. For us, $x$ is a point in space-time, and the fields take values in some other space, for example the space of a compact Lie Group. The smearing parameter $s \geq 0$ is a dimensionful quantity, a length squared in the length units of $x . S$ acts as a UV filter on the $x$-dependence, by an amount $s$. Smearing is extremely widely used in science and technology under different names of similar meaning. For a mathematical overview of linear smoothing we refer to [1].

In this write-up we shall focus on smearing in the context of gauge field theory.

\section{A brief history of smearing in gauge field theory}

Smearing is a technique in gauge theory whose scope has evolved over time. Many contributions have been made over the years and its present form is an outgrowth of these. We only mention the few instances where the scope of smearing was extended in a qualitative manner.

In classical Yang Mills theory smearing enters under the name "gradient flow" [2]. Here the fields are classical and smooth and so is the flow parameter, which we denoted by $s$ (for smearing).

In lattice field theory smearing seems to have been employed first by the APE group [3]. The new ingredient here is that smearing is completely discretized: the fields live on a lattice (no longer in the continuum) and the variable $s$ is also discretized into smearing steps. Many variants of smearing have appeared over the years within the same scope, optimizing on various features. We only mention some of the various terms that were used, in addition to "smearing": "cooling", "smoothing", "fattening", "UV filtering", "Gaussian filtering", "HYP". The smeared fields were viewed as an intermediate step to constructing operators which ultimately were thought of as complicated expressions in terms of the original lattice fields, designed to facilitate extracting some specific physical quantity. In this context smearing was viewed as a tweaking of lattice artifacts with the objective of shortening simulation time or enhancing the speed of convergence to the continuum limit. Relatively to the classical situation encountered in mathematics, the new ingredient was that the input into the smearing process became a quantum field which was fluctuating and non-smooth; it was UV cutoff dependent. One did only a finite number of discrete smearing steps and strict lattice locality was preserved. Consequently, the standard way of taking the continuum limit still applied. Two essential parameters characterized a smearing procedure: a step size and a number of steps. It was physically clear however, that only their product should really matter.

The scope of smearing was further enlarged by studying the perturbative expansion of smeared lattice operators, including their dependence on the smearing details [4]. This work clarified how the total amount of smearing (step size times number of steps) becomes a continuous parameter of dimension length squared. Thus, the smearing flow parameter became smooth again, just like in the classical case, but space-time remained discrete and the UV cutoff remained explicit. Perturbation theory for fat links is described in section 15 of [5].

The last time the scope of smearing was enlarged seems to be in [6]. In section 4.1 in that paper the explicit UV cutoff was dropped also, going back to the classical situation in the sense 
that smearing was now a procedure completely defined within continuum gauge field theory. The main observation was that the renormalization of smeared observables presented none of the new divergences associated with composite operators in the traditional, unsmeared, setting:

Standard renormalization produces finite Greens functions for products of elementary fields at distinct space-time points. By "elementary fields" one means the fields that appear explicitly in the Lagrangian. As is well known, renormalization needs to be employed again when one wants to consider Greens functions which include local operators composed of elementary fields and their derivatives at the same space-time point. Depending on the complexity of the operator, a large number of arbitrary finite parts will be required to end up with well defined expressions. This issue is particularly relevant in a gauge theory, where all physical operators are composite since gauge invariance is better viewed as a redundancy of the elementary-field representation and the concept of an "elementary field" is not physical. For example, the classic choice for a complete set of physical operators is the collection of all Wilson loops. Their renormalization is well understood and the various standard ways to define finite correlation functions of Wilson loops ultimately obscure the basic fact that parallel transport round a closed curve ought to have a monodromy represented by an element of a compact group.

Continuous smearing eliminates these problems at the expense of introducing a smearing parameter. The classic renormalization of the elementary fields is enough to ensure finiteness and uniqueness of all smeared Wilson loops, so long as the smearing parameter is nonzero.

With this it seems that the circle has been closed, and classical smearing has been fully extended to the quantum level. Considering a product of smeared composite operators, each at its own positive smearing parameter, the well known structures of the operator product expansion will emerge in a limit where some of the smearing parameters are taken to zero. In the case of a Wilson loop, one can lift the loop to $R^{4} \times R_{+}$, with a continuously varying smearing parameter.

Just as the correlation functions of elementary fields in ordinary field theory are defined (up to well understood ambiguities) by the Lagrangian, one may consider smeared correlation functions of all smeared fields, whether composite or not, also as well defined: One is free to supplement the classical Lagrangian by any quantization procedure one chooses and then calculate the same smeared correlation functions one would in any other procedure. A natural framework in the context of smearing is Parisi-Wu quantization, which employs the Langevin equation. Quantum fluctuations are induced in this case by a white noise (a regularization can be introduced by controlling the high momentum modes of the noise), which abstracts the interaction with a heat bath. Without smearing, one waits until thermal equilibration and then extracts correlations. Smearing means that after equilibration one takes the heat bath temperature abruptly down to zero, and then waits some more, specifically, an amount of Langevin-time equal to the smearing parameter $s$, before extracting correlation functions. During the cooling interval of Langevin-time extent $s$, the noise is set to zero.

\section{Smearing of gauge theories}

We now get very specific to the gauge theory case. Smearing limits the resolution at which we describe physics, by a small length scale $\sqrt{s}$. This length scale is finite in physical units, say, the inverse of the square root of the string tension. The string tension is defined in the absence of 
smearing. The limitation on resolution must preserve gauge invariance. In equations this means

$$
S\left[A^{\omega}\right]=\{S[A]\}^{\omega}, \forall\{A, \omega\},
$$

where $\omega$ is the infinitesimal local gauge transformation applied to the connection $A$. In perturbation theory gauge invariance can be replaced by BRST invariance. This also works on the lattice [7]. The BRST formalism does not really need a Lagrangian: the equations of motion are sufficient. Of course, they have to be gauge covariant. This means that smearing does not have to be introduced at the Lagrangian level; we shall use an equation of motion that does not follow from a Lagrangian. The lowest order partial differential equation obeying all symmetries with smearing included is [6]:

$$
F_{\mu, s}=D_{v} F_{\mu, v}
$$

In terms of number of derivatives, the above equation is a minimal form of smearing, which indicates universality. The equation is taken on $R^{4} \times R_{+} . R^{4}$ can be replaced by any compact manifold. The first order nature in terms of $s$ requires a single boundary condition which is taken as

$$
A_{\mu}(x ; 0)=B_{\mu}(x)
$$

where the boundary field $B$ is the ordinary quantum field on $R^{4}$. Thus, the quantum fluctuations are in full strength at $s=0$ but as $s$ increases, the limitation on the resolution limits their impact on the smeared observables.

The noncompact nature of the $R_{+}$component strongly suggests a standard gauge choice: $A_{s}=$ 0 . In this gauge the gauge field smearing equation is brought to standard form. In terms of the general definition of smearing given earlier our "minimal" equation of motion set the functional $F[$.$] to -\frac{\delta \mathbf{A}[A]}{\delta A_{\mu}(x)}$, where $\mathbf{A}$ is the classical Yang Mills action. One advantage of this choice for $F[$.$] is that the original Lagrangian of the theory also defines the smearing procedure. Hence, any$ regularization that is defined at the Lagrangian level (like, for example, a lattice or a Pauli-Villars regularization) naturally produces a smearing procedure.

\section{Smeared Wilson loops}

Introduce a loop operator $\Psi(\mathscr{C}, x, s)=\mathscr{P} e^{i g_{0} \oint_{\mathscr{C}, x}^{x} A_{\mu}(x, s) d x_{\mu}}$. The Wilson loops are $W_{r}[\mathscr{C}, s]=$ $\left\langle\chi_{r}[\Psi(\mathscr{C}, x, s)]\right\rangle$ with $r$ an irreducible representation and $s>0$. We use the normalization $\chi_{r}(\mathbf{1}) \equiv 1$. $g_{0}$ is the bare coupling constant. $\mathscr{C}$ is a smooth closed curve and $x$ is a point on it. $A_{\mu}(x, s)$ is the smeared gauge field defining a traceless hermitian matrix valued one form on $R^{4}$, smoothly parameterized by $s$.

For any $s>0$, after the introduction of the standard counter terms, the calculation of $W_{r}$ produces no new UV divergences. Consequently, $\Psi$ can be thought of as a fluctuating $S U(N)$ matrix, strictly obeying the constraints $\Psi^{\dagger} \Psi=1$ and $\operatorname{det} \Psi=1$. This remains true beyond perturbation theory.

Other regularizations of $W_{r}$ tend to violate the bound $\left|W_{r}\right| \leq 1$ which would follow from $\Psi$ being a fluctuating unitary matrix. This happens because the ordinary Coulomb term enters with a plus sign in the exponent and the negative perimeter divergence is thrown out. Smearing, on the other hand, regulates away the singularity of the Coulomb potential at short distance and replaces 
the negative divergent perimeter term by a negative term with a $\frac{1}{\sqrt{s}}$ dependence. So far, smeared Wilson loops seem to be the single option available to maintain the $S U(N)$-matrix character of the quantum variable $\Psi$ simultaneously in perturbative and non-perturbative quantum gauge theory Moreover, $W_{r}[\mathscr{C}, s]$ is a re-parameterization and zig-zag invariant Stokes functional of $\mathscr{C}$. Later, we shall argue that if there exists, in some sense, a dual string representation of $W_{r}[\mathscr{C}, s]$ for $s=0$, there should exist one also for $s>0$.

We first restrict our attention to curves that are continuous, have a continuous tangent and do not self-intersect. Define couplings $g_{w}^{2}$ by $\log \left[W_{r}[\mathscr{C}, s]\right]=-g_{w}^{2}[\mathscr{C}, s, r] C_{2}(r) f_{\text {tree }}[\mathscr{C}, s]$, where $f_{\text {tree }}$ is defined by tree level perturbation theory, when $g_{w}^{2} \rightarrow g_{0}^{2}$. We parameterize the curves by an overall scale $l$, the perimeter of the curve, and a set of shape variables $\zeta_{\alpha} . \mathscr{C}$ now refers to all curves that are translations and rigid rotations of the same curve. The set $\zeta_{\alpha}$ is invariant under dilatations. The entire dependence on dilatations resides in $l$. The function $f_{\text {tree }}$ depends only on the $\zeta_{\alpha}$ and on $\frac{l}{\sqrt{s}}$. For $l, \sqrt{s} \ll \Lambda_{N}^{-1}$, fixed $\zeta_{\alpha}$ and $\frac{l}{\sqrt{s}}$, we can calculate the perturbative running of $g_{w}^{2}$ as a function of $l$.

$f_{\text {tree }}$ is a functional of $\mathscr{C}$ already defined in abelian Yang Mills theory. Assuming a fixed amount of smearing, one has a short distance expansion for $l \rightarrow 0$ and a long distance one for $l \rightarrow \infty$ : The long distance expansion, for $l \rightarrow \infty$, gives:

$$
f_{\text {tree }}[\mathscr{C}, s]=a_{0}[\mathscr{C}] \frac{l}{\sqrt{s}}+a_{1}[\mathscr{C}]+a_{2}[\mathscr{C}] \frac{\sqrt{s}}{l}+\ldots
$$

At tree level the theory is conformal, and therefore the real expansion parameter is the ratio $\frac{l}{\sqrt{s}}$, so large loops of a given shape correspond to some fixed size loop of the same shape, but at high resolution.

$a_{0}[\mathscr{C}]$ equals $\frac{1}{8 \pi \sqrt{2 \pi}}$, providing the coefficient of what would become the perimeter divergent term when $s \rightarrow 0$ in the abelian case. $a_{1}[\mathscr{C}]$ only depends on the $\zeta_{\alpha}$ and is proportional to the "number of photons" first introduced (with different motivation) by L. Stodolsky [8]:

$$
a_{1}[\mathscr{C}]=\frac{1}{4 \pi^{2}} \oint d x_{\mu} \oint d y_{\nu}\left[\frac{\delta_{\mu v}}{(x-y)^{2}}-\frac{(x-y)_{\mu}(x-y)_{v}}{(x-y)^{4}}\right] .
$$

The integrand in the above expression is the photon propagator in a particular gauge, but, going from that gauge to Feynman gauge for example, involves a gauge function that is singular on the loop. This is how the above expression, which is finite, becomes formally gauge equivalent to an expression that has a perimeter divergence. The same manipulation in the smeared case is valid, as the singularity is smeared away. Thus, smearing provides yet another way to arrive at Stodolsky's formula, by explicitly removing the term $a_{0}[\mathscr{C}] \frac{l}{\sqrt{s}}$ and taking $s \rightarrow 0$ afterwards. Moreover, since $g_{w}^{2}[\mathscr{C}, s, r]$ is well defined in the fully interacting nonabelian quantum field theory, the long distance expansion of $g_{w}^{2}$ provides a possible extension of Stodolsky's photon number to the non-abelian case.

Similarly, the short distance expansion, $l \rightarrow 0$, looks like

$$
f_{\text {tree }}[\mathscr{C}, s]=b_{0}[\mathscr{C}] \frac{l^{4}}{s^{2}}+b_{1}[\mathscr{C}] \frac{l^{6}}{s^{3}}+\ldots
$$

Here,

$$
b_{0}[\mathscr{C}]=\frac{1}{2^{9} \pi^{2}} \frac{\sum_{\mu \nu} \sigma_{\mu, v}^{2}}{l^{4}}
$$


where $\sigma_{\mu, v}=\frac{1}{2} \oint\left(d x_{\mu} x_{v}-d x_{v} x_{\mu}\right)$ is the enclosed area of the projection of $\mathscr{C}$ on the $\mu, v$ plane. In this case the expectation value defining $g_{w}^{2}$ is $\operatorname{Tr}_{f}\left\langle F_{\mu \nu}^{2}(x, s)\right\rangle$. At one higher order we get terms of the form $\left\langle(D F)_{\mu \nu}^{2}(x, s)\right\rangle$, etc.

The structure of this short distance expansion extends from the tree level all the way beyond perturbation theory. A short distance expansion of Wilson loops was discussed in [9]. There the associated condensates were described employing a physically sensible, albeit imprecise, concept of separation between perturbative and nonperturbative contributions. Smearing provides one possible disambiguation of this description.

If we allow isolated discontinuities in the tangent to the curve there are logarithmic corner divergences when $s \rightarrow 0$, locally associated with each discontinuity in the tangent.

For specific simple loop shapes one can calculate $f_{\text {tree }}[\mathscr{C}]$ explicitly. For example, consider a circle of radius $R$. We have, with $\rho=\frac{2 s}{R^{2}}$,

$$
f_{\text {tree }}(\text { circle })=-\frac{1}{4}+\frac{e^{-\frac{1}{2 \rho}}}{8 \rho}\left[(1+2 \rho) I_{0}\left(\frac{1}{2 \rho}\right)+I_{1}\left(\frac{1}{2 \rho}\right)\right],
$$

where the $I_{n}($.$) are modified Bessel functions.$

For a rectangular $T \times R$ loop, we get a more complicated expression in terms of an integral with an error function inside the integrand. In an asymptotic expansion for large loops one recovers the would be linear and logarithmic divergences. Beyond that, one has domination by the Coulomb term, linear in $\frac{R}{T}+\frac{T}{R}$. Such a term also appears in an effective string representation expected to be valid for large rectangular loops. There it no longer is multiplied by a coupling constant, but rather by a universal number. This gives an indication how one might be able to match perturbative to string expressions.

For numerical purposes, $f_{\text {tree }}$ has also been calculated on the lattice with a single plaquette Wilson action.

\section{Smearing in loop calculus}

As is well known, one expects Yang Mills theory with gauge group $S U(N)$ to be related to some string theory with a string coupling going to zero as $N \rightarrow \infty$. Thus, at least in the large- $N$ limit, one does not need a completely well defined string theory; just a well defined generalized two dimensional sigma-model would do, if it were the right one. To be sure, the observable we are interested in in this two dimensional field theory is a complicated loop functional, so even if somebody handed us the right sigma-model we still would face a difficult problem. At $N=\infty$ the set of field-theoretical Schwinger Dyson equations for Wilson loops closes on loops in the fundamental representation, producing the Makeenko-Migdal [10] loop equation. It should be solved by the loop functional coming from the sigma-model. The loop equation is elegant only at a formal, unregularized level. In the smeared setup, it is a property of the initial condition, expressed in terms of the boundary fields $B$. The attractiveness of the equation stems from the fact that all the Yang Mills structure has disappeared, being replaced by a partial differential equation on loop space. More specifically, there appears a loop Laplacian, whose definition is relatively well understood. The solution to the equation should be a member of a specific subset of all possible 
loop functionals: it must be of "Stokes" type, be reparameterization invariant and obey a zig-zag symmetry.

The above properties hold for smeared Wilson loops. One would therefore like to be able to write down some loop equation directly for smeared loops. This would provide an opening to make the required defining property of the dual field theoretic - string representations concrete, as it would deal with well defined objects.

Although this was not mentioned in [6], the original motivation for choosing smearing as a way to handle composite operators was that it could be represented in loop space with the help of the same loop Laplacian which appears in the Makeenko-Migdal equation:

$$
\frac{\partial W_{r}[\mathscr{C}, s]}{\partial s}=\oint d \sigma \frac{\delta^{2} W_{r}[\mathscr{C}, s]}{\delta x_{\mu}^{2}(\sigma)} .
$$

The original Makeenko-Migdal equation places a constraint on the initial data, at $s=0$. To what degree this fully determines the initial data takes us back to the unsolved problem of finding a finite, elegant version of the Makeenko-Migdal equation. Nevertheless, we can say that, if indeed a string route is found which makes sense of the original Makeenko-Migdal equation, this route would also make sense of a smeared version of the solution to these equation and therefore smearing should extend to the string theory. Alternatively, another view of the Makeenko-Migdal equation in the smeared context is as an asymptotic condition at $s \rightarrow 0$ on a solution of the loop-space heat equation describing smearing.

\section{Hamiltonian smearing}

Smearing as defined above will obscure constraints on correlation functions which represent Minkowski space unitarity. It is possible to avoid this by smearing only in the space directions.

\section{References}

[1] I. J. Schoenberg, “On smoothing smoothing operations and their generating functions", Bull. Amer. Math. Soc. vol. 59, 199 (1953).

[2] M. F. Atiyah and R. Bott, "The Yang-Mills equations over Riemann surfaces”, Phil. Trans. R. Soc. Lond. A 308, 523 (1982); S. K. Donaldson, “Anti self-dual Yang-Mills connections over complex algebraic surfaces and stable vector bundles”, Proc. London Math. Soc. (3) 50, 1 (1985).

[3] M. Albanese et al. [APE Collaboration], Phys. Lett. B 192, 163 (1987).

[4] C. Bernard, T DeGrand, Nucl. Phys. B - Proc. Suppl. 845 (2000).

[5] S. Capitani, Physics Reports 382, 113 (2003).

[6] R. Narayanan, H. Neuberger, JHEP03, 064 (2006).

[7] H. Neuberger, Nucl. Phys. B175, 69 (1986).

[8] L. Stodolsky, Acta Phys. Pol. B 33, 2659 (2002).

[9] M. A. Shifman, Nucl. Phys. B173, 13 (1980).

[10] Y. Makeenko, "Methods of Contemporary Gauge Theory", Cambridge Monographs on Mathematical Physics (2002). 